\title{
Clinical Features and Genetic Analysis of 20 Chinese Patients with X-Linked Hyper-IgM Syndrome
}

\author{
Lin-Lin Wang, ${ }^{1}$ Wei Zhou, ${ }^{2}$ Wei Zhao, ${ }^{1,3}$ Zhi-Qing Tian, ${ }^{1}$ Wei-Fan Wang, \\ Xiao-Fang Wang, ${ }^{1}$ and Tong-Xin Chen ${ }^{1,2,4}$ \\ ${ }^{1}$ Department of Allergy and Immunology, Shanghai Children's Medical Center, Shanghai Jiao Tong University School of Medicine, \\ Shanghai 200127, China \\ ${ }^{2}$ Department of Nephrology and Rheumatology, Shanghai Children's Medical Center, Shanghai Jiao Tong University School of Medicine, \\ Shanghai 200127, China \\ ${ }^{3}$ Division of Allergy and Immunology, Department of Pediatrics, Virginia Commonwealth University, Richmond, VA 23298, USA \\ ${ }^{4}$ Division of Immunology, Institute of Pediatric Translational Medicine, Shanghai Jiao Tong University School of Medicine, \\ 1678 Dongfang Road, Shanghai 200127, China
}

Correspondence should be addressed to Tong-Xin Chen; tongxinc@sjtu.edu.cn

Received 27 May 2014; Accepted 31 July 2014; Published 20 August 2014

Academic Editor: Catharina Schuetz

Copyright (C) 2014 Lin-Lin Wang et al. This is an open access article distributed under the Creative Commons Attribution License, which permits unrestricted use, distribution, and reproduction in any medium, provided the original work is properly cited.

$\mathrm{X}$-linked hyper-IgM syndrome (XHIGM) is one type of primary immunodeficiency diseases, resulting from defects in the CD40 ligand/CD40 signaling pathways. We retrospectively analyzed the clinical and molecular features of 20 Chinese patients diagnosed and followed up in hospitals affiliated to Shanghai Jiao Tong University School of Medicine from 1999 to 2013. The median onset age of these patients was 8.5 months (range: 20 days-21 months). Half of them had positive family histories, with a shorter diagnosis lag. The most common symptoms were recurrent sinopulmonary infections (18 patients, $90 \%)$, neutropenia (14 patients, $70 \%)$, oral ulcer (13 patients, 65\%), and protracted diarrhea (13 patients, 65\%). Six patients had BCGitis. Six patients received hematopoietic stem cell transplantations and four of them had immune reconstructions and clinical remissions. Eighteen unique mutations in CD40L gene were identified in these 20 patients from 19 unrelated families, with 12 novel mutations. We compared with reported mutation results and used bioinformatics software to predict the effects of mutations on the target protein. These mutations reflected the heterogeneity of CD40L gene and expanded our understanding of XHIGM.

\section{Introduction}

X-linked hyper-IgM syndrome (XHIGM; HIGM1; OMIM: 308230) is one type of primary immunodeficiency diseases (PIDs), resulting from defects in the CD40 ligand/CD40 signaling pathways leading to impairment of immunoglobulin isotype switching in B cells and characterized by recurrent infections in association with markedly decreased serum $\operatorname{IgG}$, IgA, and IgE levels but normal or elevated serum IgM levels [1]. Patients with XHIGM usually develop symptoms by the first or second year of life. Over $50 \%$ of patients have chronic or intermittent neutropenia, often associated with oral ulcers. Opportunistic infections, especially Cryptosporidium parvum and Pneumocystis jirovecii infections, are common and prominent clinical feature of the patients. Besides the susceptibility to recurrent bacterial and opportunistic infections, these patients are prone to autoimmune manifestations, especially hematologic abnormalities, arthritis, and inflammatory bowel disease. Furthermore, an unusual susceptibility to liver, pancreas, or biliary tract tumours has been reported [2].

CD40L is the disease-causing gene of XHIGM [3-7], located in Xq26.3-Xq27.1 and containing five exons and four intervening introns. The corresponding translated protein CD154 (also called CD40L, gp39, TNFSF5, or TRAP) contains 261 amino acids and is mainly expressed on activated mature $\mathrm{T}$ cells. Its receptor, $\mathrm{CD} 40$, is expressed on a variety of cells, such as B cells, monocytes/macrophage, dendritic cells, endothelial cells, and epithelial cells $[8,9]$. CD40-CD154 interactions provide a costimulatory signal for 
$\mathrm{T}$ cell activation and can lead to $\mathrm{B}$ cell proliferation and immunoglobulin class switching, affecting both cellular and humoral immunity $[10,11]$. Therefore, XHIGM belongs to a combined immunodeficiency disease category.

XHIGM is the most common subtype of hyper-IgM syndromes (affecting about $70 \%$ of the patients) [12]. More subtypes of hyper-IgM syndromes have been gradually revealed. Diseases resulting from mutations in AICDA (ID: 57379), CD40 (ID: 958), UNG (ID: 7374), NEMO (ID: 8517), or NFKBIA (ID: 4792) genes are all involved in the CD154-CD40- NF- $\kappa$ B pathways and affected the class switch recombination (CSR), somatic hypermutation (SHM), or germinal center formation and form the subtypes of hyperIgM syndromes. Mutations in the promoter of $C D 40 \mathrm{~L}$ were also found [13]. The heterogeneity of hyper-IgM syndrome brings the challenge for diagnosis and accurate and reliable molecular testing methods are needed.

With diagnosed patients accumulated, people began to analyze the clinical features and mutation characteristics of these patients since 1992 [14]. A CD40L mutation database (http://structure.bmc.lu.se/idbase/CD40Lbase/index.php) has been founded and updated [15]. Retrospective research results of XHIGM patients in Europe [16], USA [17], Japan [18], and Latin America [19] were published. However, little information about Chinese XHIGM patients has been reported. In this study, we collected clinical data of Chinese XHIGM patients diagnosed and followed up in our center, reviewed their clinical and immune features, therapy, and response, and investigated the mutation characteristics, intending to increase our knowledge of this disease and finally improve the quality of life in these patients.

\section{Methods}

2.1. Patients. Patients in present study were first diagnosed in hospitals affiliated to Shanghai Jiao Tong University School of Medicine or referred from other hospitals and followed up in Shanghai Children's Medical Center (also affiliated to Shanghai Jiao Tong University School of Medicine) during 1999-2013. A total of 28 candidate patients with HIGM phenotype were recruited. The inclusion criteria were low sera IgG and IgA ( 2 standard deviations below normal value for age), normal or elevated serum IgM, and compatible infectious events. Patients with secondary immunodeficiency conditions such as HIV or congenital rubella infections, immunosuppressive drug use, or neoplasms were excluded. Then CD154 expression on active $\mathrm{T}$ cells was detected by flow cytometry and sequences of CD40L gene were analyzed. A total of 20 patients with XHIGM were finally identified.

2.2. Data Collection. An informed consent was obtained from each patient's parent or guardian before enrollment in the study. Clinical data were collected from the patients' medical records, including initial clinical manifestation, onset age, diagnosis age, parental consanguinity, family history of immunodeficiency, recurrent infections, autoimmune diseases or malignancy diseases, vaccination and allergic history, complication, laboratory tests (including immunoglobulin level and lymphocyte subpopulations), and treatments. A total of $5 \mathrm{~mL}$ venous blood was collected from patients for pathogen screening and gene sequencing.

2.3. Molecular and Genetic Diagnosis. Detection of CD154 expression on activated CD4+ T cells was performed by flow cytometry (FACScan, Becton Dickinson, USA) using specific fluorescent-labeled monoclonal antibodies according to the method described previously [20].

Genomic DNA was isolated from heparinized peripheral blood by using the DP319 kit (Tiangen Biotech Co. LTD., China). The individual exons of CD40L gene, including exon-intron boundaries, were amplified by polymerase chain reaction (PCR) as previously described [21]. The system for PCR reaction contained $13.5 \mu \mathrm{L} \mathrm{dd}_{2} \mathrm{O}, 1 \mu \mathrm{L}$ primer for each direction, and $8.5 \mu \mathrm{L} 2 \times$ Taq PCR MasterMix (containing $0.1 \mathrm{UTaq} / \mu \mathrm{L}, 500 \mu \mathrm{M}$ dNTP, $20 \mathrm{mM}$ Tris-HCl ( $\mathrm{pH} 8.3$ ), $100 \mathrm{mM} \mathrm{KCl}$, and $3 \mathrm{mM} \mathrm{MgCl} 2$ (Tiangen Biotech Co. LTD., China), with total volume $25 \mu \mathrm{L}$ ). Amplification conditions were $95^{\circ} \mathrm{C}$ for 10 minutes, then 40 cycles of $94^{\circ} \mathrm{C}$ for 30 seconds, $55^{\circ} \mathrm{C}$ for one minute, and $72^{\circ} \mathrm{C}$ for 30 seconds, followed by a final extension of $72^{\circ} \mathrm{C}$ for 2 minutes. The PCR products were electrophoresed in 1.5\% agarose gel, purified, and sequenced. Mutations were investigated by alignment with standard sequence published in NCBI. We sequenced CD40L genes in 50 Chinese controls. Human SNP databases (dbSNP in NCBI and 1000 Genomes) were also searched to confirm the detected mutations.

2.4. Analysis Tools. Data were analyzed by SPSS statistical software (version 21.0, SPSS Inc., Chicago, IL). Median and range were used to present the characters of focused variable. Bioinformatics software PolyPhen-2 (http://genetics.bwh .harvard.edu/pph2/) and MutationTaster (http://www.mutationtaster.org/) were used to predict the effects of point mutations.

\section{Results}

3.1. Demographic Characteristics. From 1999 to 2013, 20 patients from 19 unrelated families (P2 and P3 are cousins) were diagnosed as XHIGM, considering the clinical manifestations, lab examination, family history, decreased CD154 expression, and the mutation of CD40L gene (Table 1). The median onset age of these patients is 8.5 months (range: 20 days-21 months). And the median of diagnosis lag is 50 months (range: 5 months -15 years). Half of the patients had positive family histories of previous sibling deaths at an early age and the diagnosis lag in them was much shorter than that in patients with negative family histories $(31 \pm 23$ months versus $85 \pm 55$ months, $P=0.015$ ). Positive family history with early death in male members may help in diagnosis.

3.2. Clinical Manifestations. The most common symptoms of XHIGM patients were recurrent sinopulmonary infections (18 patients, $90 \%$ ), neutropenia (14 patients, $70 \%$ ), oral ulcer (13 patients, 65\%), and protracted diarrhea (13 patients, $65 \%)$. Respiratory and gastrointestinal systems were more 


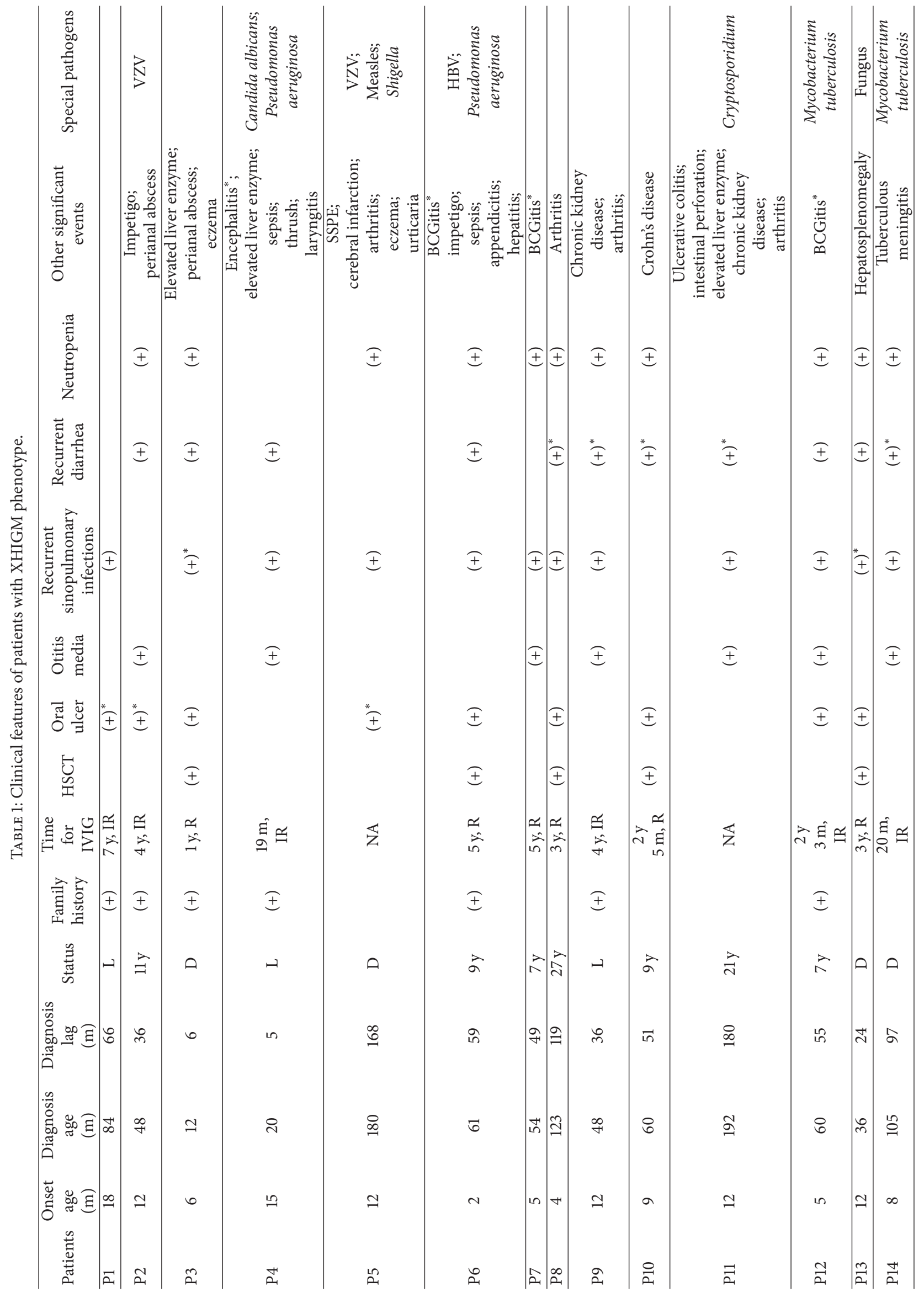




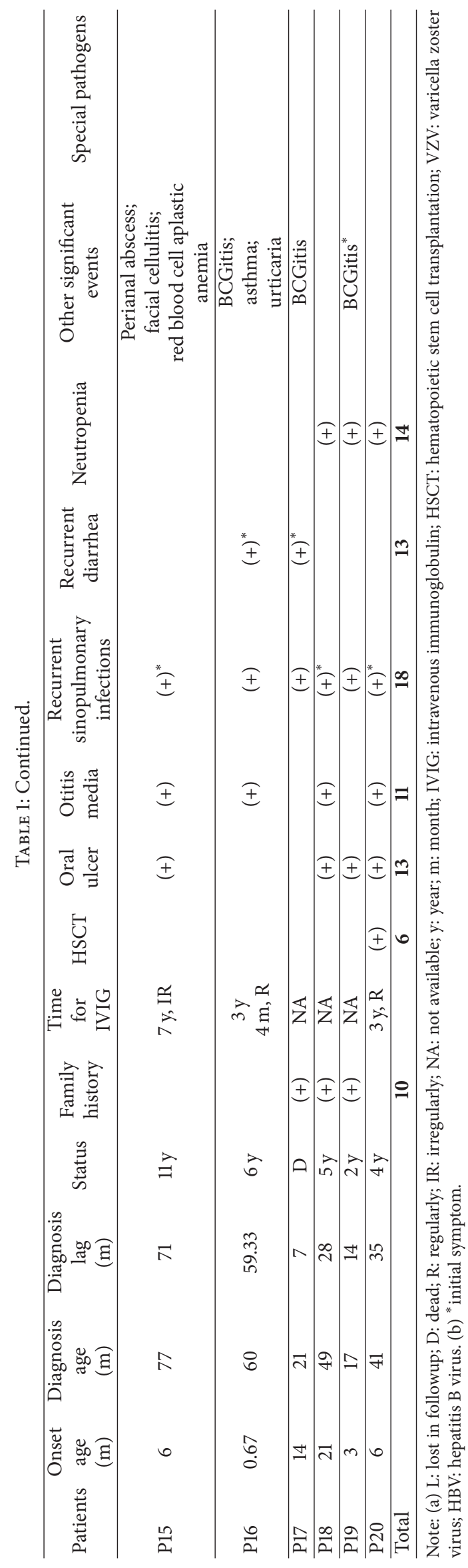


frequently affected. Skin (7 patients, 35\%), bone and joint system (4 patients, 20\%), central nervous system (3 patients, $15 \%)$, and urinary system ( 2 patients, $10 \%$ ) were also affected (detailed in Table 1).

The median onset age of diarrhea was 12.0 months (range: 20 days to 20 months). Among them, two patients (P10, P11) were diagnosed with inflammatory bowel diseases (IBDs) under enteroscopes, and Cryptosporidium was isolated from the stool of P11. Fungus was isolated from the stool of P13. Pathogens in other patients were not identified.

Six patients had BCGitis (Table 2). The median onset time of adverse drug reactions was 2 months old (range: 50 days-7 months). Two of the patients received antituberculosis medicine treatments and recovered. The other four patients had severe local adverse events after BCG vaccinations, presenting ulceration $>10 \mathrm{~mm}$ or suppurative lymphadenitis, and received surgeries to drain. Among them, P12 was reinfected by tubercle bacillus at 35 months old and recovered after antituberculosis drug therapy. Besides, another patient P14 suffered pulmonary tuberculosis at 8.5 years old, with restrictive pulmonary capacity and splenomegaly and lymphadenopathy. The patient received isoniazide and rifampicin treatment unregularly for nearly one year and developed tuberculous meningitis and died at 10 years old.

Some special manifestations deserved extra attention. P5 was infected by measles and developed subacute sclerosing panencephalitis (SSPE). P11 had Cryptosporidium infection, with protracted diarrhea and elevated liver enzyme. Moreover, this patient developed chronic kidney disease, presenting oliguria, edema, acidosis, increased creatinine concentration, and decreased glomerular filtration rate (GFR), lasting for over four months. Renal biopsy showed tubulointerstitial lesions and the patient received dialysis. P9 also developed chronic kidney disease, with oliguria, albuminuria, hematuria, increased serum creatinine and urea nitrogen concentration, and anemia for over three months. He also exhibited electrolyte disturbance (hypocalcemia and hyperphosphatemia) and convulsion. Ultrasound examination revealed diffuse kidney impairments.

3.3. Hematologic Findings. Anemia was found in seven patients (Table 3). Among them, P10 and P11 had IBDs and the chronic bloody purulent stools might partly cause the anemia. P9 and P11 had chronic kidney disease which may lead to renal anemia. P15 had pure red blood cell aplastic anemia demonstrated by bone marrow biopsy. Neutropenia was the most common hematologic complication, occurring in $70 \%$ of the patients (14 patients), 11 of which complicated with oral ulcer.

The median serum IgM concentration was $3.15 \mathrm{~g} / \mathrm{L}$ (range: $0.74-11.7 \mathrm{~g} / \mathrm{L}$ ). Seventeen patients had elevated IgM levels and three patients had normal IgM levels. Serum IgG levels were uniformly decreased. IgA levels were reduced in the majority of patients.

Lymphocyte counts were generally normal. Three patients (P5, P9, and P11) exhibit significantly decreased CD4/CD8 ratio. They all had severe clinical symptoms and the decreased ratio reflected the impaired cellular immunity.
P5 had measles infection, leading to SSPE, and died within one year. P11 had Cryptosporidium infection, with prolonged elevated liver enzyme and impaired renal functions. P9 also had severe manifestation, complicated with chronic renal failure.

3.4. Mutation Analysis. Eighteen unique mutations were identified in 20 patients from 19 unrelated families (P2 and P3 were cousins and they had the same mutation. Besides, P12 and P16 had the same mutation). Among the 19 families, we found 7 deletions, 6 missense mutations, 2 nonsense mutations, 2 splice site mutations, and 2 insertions (Table 4). The mutations were distributed throughout the entire gene, affecting all the domain of the protein, and exon 5 was the most frequently affected region (10/19, 52.6\%). Detailed descriptions and analysis were in the Discussion section.

3.5. Therapy and Prognosis. Five patients had died (Table 1). P5 developed SSPE after measles virus infection at 15 years old and died within one year. P14 was infected by mycobacterium tuberculosis and died of tuberculous meningitis at 10 years old. P17 had severe diarrhea and pneumonia since 14 months and died at 2 years old. P3 and P13 received hematopoietic stem cell transplantations (HSCT) but suffered from severe infections and died at 22 months and 56 months, respectively. Three patients were lost in followups. The other twelve patients were alive till now, with the median age of 8 years old (range: 2-27 years old).

Fifteen patients $(75 \%)$ received intravenous immunoglobulin (IVIG) treatment (Table 1). Eight patients could receive IVIG regularly ( $400 \mathrm{mg} / \mathrm{kg} / \mathrm{month}$ ) after diagnosis as HIGM. All of them were free of severe bacterial infections, although three of them still had recurrent oral ulcers. Seven patients could not receive full-dose IVIG regularly. Among them, one patient (P14) died of tuberculosis infection. Two patients still had recurrent bacterial infections (P2 had recurrent otitis media and perianal abscess. P12 had otitis media, pneumonia, and fever every half month). Three patients were lost in followup. The other five patients did not receive IVIG therapy, including the two patients who died from measles infection (P5) and severe diarrhea and pneumonia (P17).

Six patients received HSCTs and the median age at transplantation was 6 years old. Four patients received hematopoietic stem cells from HLA-identical unrelated donors and they had clinical remissions and were followed up from months to 6 years. The other two patients had HLA-half identical sibling donors and died from severe infections (Table 5).

\section{Discussions}

This study described the clinical features of Chinese XHIGM patients. Respiratory and gastrointestinal systems were more frequently affected. Skin, bone and joint system, central nervous system, and urinary system were also involved. In this study, two patients (P9 and P11) developed chronic kidney diseases. Cardiovascular disease, infections, and nonsteroidal anti-inflammatory drugs (NSAIDs) are accepted risk factors for causing chronic kidney disease [22]. P9 was born with 
TABLE 2: BCG vaccination-induced adverse drug reactions (ADR) and mycobacterium infections in XHIGM patients.

\begin{tabular}{|c|c|c|c|}
\hline Patients & Ages & Performances & Treatments \\
\hline P6 & $2 \mathrm{~m}$ & BCGitis (ipsilateral axillary lymphadenitis) & Surgery \\
\hline P7 & $2 \mathrm{~m}$ & BCGitis (ipsilateral axillary lymphadenitis) & Surgery \\
\hline \multirow[b]{2}{*}{$\mathrm{P} 12$} & $50 \mathrm{~d}$ & BCGitis (ipsilateral axillary lymphadenectasis) & Antituberculosis medicine (rifampicin, unregularly) \\
\hline & $35 \mathrm{~m}$ & Pulmonary tuberculosis & $\begin{array}{l}\text { Antituberculosis medicine (isoniazide + rifampicin + VitB6), } \\
\text { antibiotics }\end{array}$ \\
\hline P16 & $7 \mathrm{~m}$ & BCGitis (ipsilateral axillary lymphadenitis) & Surgery \\
\hline P17 & $2 \mathrm{~m}$ & BCGitis (ipsilateral axillary lymphadenitis) & Surgery \\
\hline $\mathrm{P} 19$ & $3 \mathrm{~m}$ & BCGitis (ipsilateral cervical lymphadenectasis) & Antituberculosis medicine \\
\hline \multirow[t]{2}{*}{$\mathrm{P} 14$} & $8.5 \mathrm{y}$ & $\begin{array}{l}\text { Pulmonary tuberculosis, } \\
\text { restrictive pulmonary capacity, } \\
\text { splenomegaly and lymphadenopathy }\end{array}$ & $\begin{array}{l}\text { Antituberculosis medicine (isoniazide + rifampicin, unregularly), } \\
\text { antibiotics, IVIG, thymosin }\end{array}$ \\
\hline & $10 \mathrm{y}$ & Tuberculous meningitis & Antituberculosis medicine (isoniazide + rifampicin, unregularly) \\
\hline
\end{tabular}

Note: IVIG: intravenous immunoglobulin.

congenital heart diseases (ventricular septal defect and patent ductus arteriosus). P11 had ulcerative colitis and received NSAIDs treatment. Both of the patients had severe recurrent infections because of immunodeficiency. These complications might partly contribute to the kidney involvement in these two patients. Few reports on XHIGM patients with chronic kidney diseases have been published till now. We should pay more attention to the urinary system function in further followups.

Opportunistic infections, especially Cryptosporidium parvum and Pneumocystis jirovecii infections, are common and prominent clinical feature of XHIGM patients [16, 17, 23]. Besides, infections caused by tubercle bacillus were detected $[16,17,19]$. A number of PIDs are reported to be susceptible to severe mycobacterial disease following vaccination with BCG, including severe combined immunodeficiency (SCID), chronic granulomatous disease (CGD), and Mendelian susceptibility to mycobacterial diseases (MSMD). Increased BCG infections are also reported in patients with hyperimmunoglobulin E syndrome (HIES) and XHIGM, with less prevalence and severity of BCG complications compared with the PIDs mentioned above [24]. In a European survey, only two of the 56 XHIGM patients present with tuberculosis [16]. However, in our study concerning 20 XHIGM patients, we found six patients had BCGitis during the first year of life. One of them was reinfected by tuberculosis at 35 months old. One more patient suffered pulmonary tuberculosis and tuberculous meningitis and died at 10 years old. In another cohort study focusing on Chinese patients, five (38\%) of the 13 XHIGM patients had lymphoid tuberculosis after BCG vaccination [25], the relatively high incidence of which was very similar to ours. An Indian research group also reported that two patients with pulmonary tuberculosis and one patient with disseminated BCGiosis were found in the cohort study including seven XHIGM patients [26]. Global Tuberculosis Report 2013 by WHO pointed out that India and China had the largest number of tuberculosis cases, taking up to $26 \%$ and $12 \%$ of the global total, respectively [27]. In both China and India, BCG vaccine is given to every newborn after birth to prevent tuberculous meningitis and disseminated disease, while in Europe, where incidence and prevalence of tuberculosis are low, less than half of the European countries have nationally recommended BCG vaccination. The differences in tuberculosis prevalence and BCG coverage may partly contribute to the differences of mycobacteria complications in XHIGM patients.

Some recent discoveries may help to elucidate the susceptibility to mycobacterial infections in XHIGM patients. Hayashi's results indicated that CD40-CD154 signaling might be an important step in host immune response against Mycobacterium avium infection [28]. Klug-Micu et al. revealed that activation of monocytes via CD40L resulted in a Vitamin D-dependent antimicrobial activity against Mycobacterium tuberculosis [29]. IL12/23-IFN- $\gamma$ axis is involved in defending against BCG infection $[24,30]$. Jain et al. [31] demonstrated that activated $\mathrm{T}$ cells from XHIGM patients produced markedly reduced levels of IFN$\gamma$ and failed to induce antigen-presenting cells to synthesize IL-12. We should continue to observe the susceptibility to tuberculous infections in XHIGM patients and be concerned about the immunity affected by tuberculous infection. Furthermore, we found two patients with varicella-zoster virus infections, one patient with measles virus infections, one patient with fungus infections, and one patient with Cryptosporidium infection, which all indicated the defects in cellular immunity.

Mutations of CD40L geneare highly heterogeneous [18$20,32]$. CD40Lbase, which was last updated in 2011, contained 250 public entries. Besides, more mutations on CD40L gene were reported in other studies $[19,33]$. Considering the published results, the common type of mutations in CD40L might be missense mutations, followed by deletion/insertion mutations, nonsense mutations, and splice site mutations (detailed in Table 6). In our results, missense mutations and deletion/insertion mutations were the main types, composing about $3 / 4$ of the mutations.

Some possible mutation hotspots have been identified. Notarangelo et al. [15] pointed out that W140 represents a mutational hotspot. In Lee's study [32], four possible 


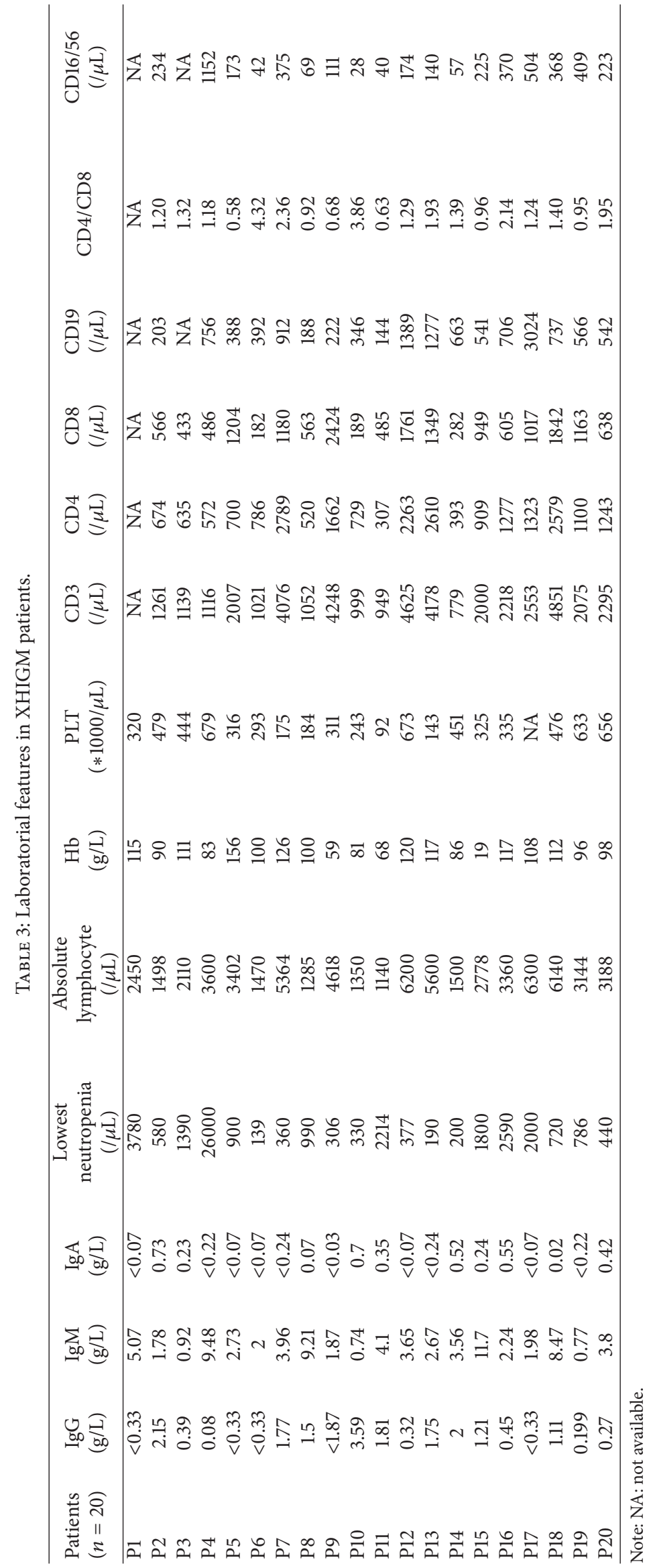




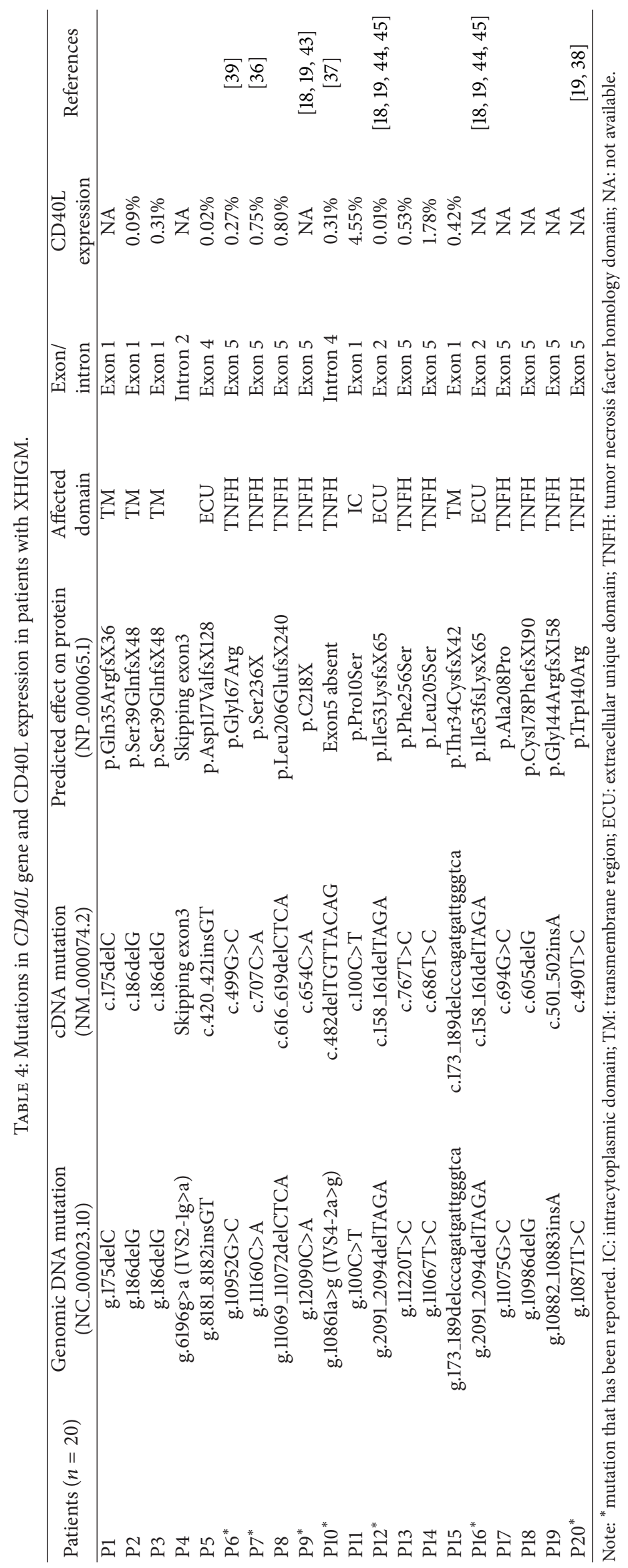




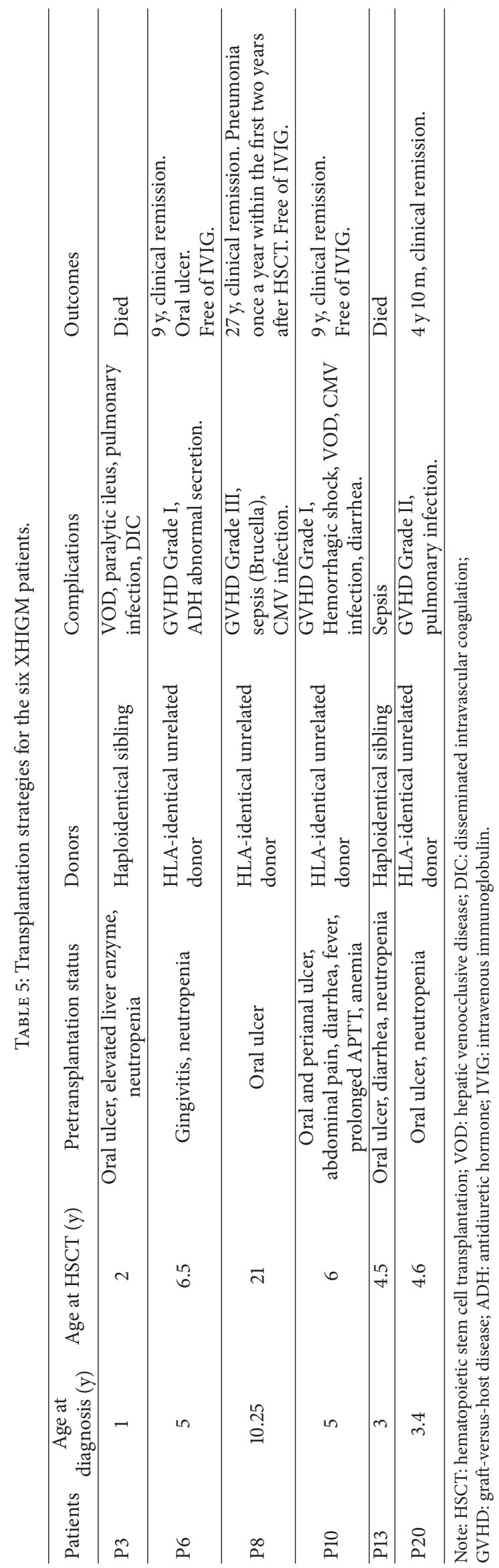


TABLE 6: Mutation types in CD40L gene.

\begin{tabular}{|c|c|c|c|c|c|c|c|}
\hline Reports & Year & Number of mutations & Missense (\%) & Nonsense (\%) & Deletion (\%) & Insertion (\%) & Splice site (\%) \\
\hline This study & 2013 & 19 & 31.6 & 10.5 & 36.8 & 10.5 & 10.5 \\
\hline Nonoyama et al. [18] & 1997 & 13 & 30.8 & 23.1 & 23.1 & 0 & 23.1 \\
\hline Seyama et al. [20] & 1998 & 28 & 32.1 & 17.9 & 7.2 & 14.3 & 32.1 \\
\hline Lee et al. [32] & 2005 & 61 & 18.0 & 24.6 & 21.3 & 11.5 & 24.6 \\
\hline Winkelstein et al. [17] & 2003 & 54 & 22.2 & 18.5 & 24.1 & 11.1 & 24.1 \\
\hline CD40Lbase [46] & 2011 & 221 & 31.7 & 23.5 & 17.2 & 10.4 & 16.3 \\
\hline Cabral-Marques et al. [19] & 2014 & 26 & 34.6 & 26.9 & 11.5 & 7.7 & 11.5 \\
\hline
\end{tabular}

hotspots were identified that were Thr254, IVS1+1 g >t, IVS3+1 $\mathrm{g}>\mathrm{a} / \mathrm{t}$, and IVS4+1 $\mathrm{g}>\mathrm{c}$. In the CD40Lbase, mutations were concentrated in some special positions; that is, mutations in C218, T254, and W140 have been identified in more than 10 patients, respectively, till now.

In this study, we identified two splice site mutations (P4: IVS2-1 g>a and P10: IVS4-2 a>g). Vargas-Hernández et al. [34] and García-Pérez et al. [35] separately reported a IVS3-1 g>a mutation, resulting in exon 4 skipping and a protein with 21-amino acid truncation. Weller et al. [36] reported an IVS2-1 g>t mutation, resulting in exon 3 skipping and forming a protein consisting of 107 amino acids. In our study, P4 mutated in $3^{\prime}$-acceptor splice sites, the same positions as the three cases, and it can be inferred that this mutation could also result in the skipping of exon 3. For the mutation in P10, it has been reported in 1994 [37]. Mutation in the acceptor site of intron 4 allowed utilization of a cryptic acceptor site and a few base pairs downstream in exon 5 and led to 8-nucleotides deletion in P10.

Six patients had missense mutations in this study. From data published in CD40Lbase (updated to 2011), the most frequent amino acid substitutions were Leu to Ser/Pro, Thr to Met, Gly to Arg, and Ala to Asp/Glu. Thusberg and Vihinen [38] reported that there were 10 invariant positions corresponding to the amino acids W140, L161, G167, Y169, Y172, L205, G226, G227, L231, and G257 in CD40L, according to sequence alignment and calculation. Disease causing mutations are typically located at conserved positions within a protein family, since these positions are usually essential for the structure and/or function of the protein [38]. In our study, the mutation in P6 (Gly167Arg) was previously reported in two Iranian patients [39]. Substitution from glycine to arginine introduced a larger side chain group and a positive charge, which would profoundly change structure and functions of the protein. The mutation in P20 (p.Trp140Arg) was reported as one of the "hotspots" and eleven patients in all had been reported to have this mutation until now. Trp140 is a large hydrophobic residue buried inside the protein while arginine had a large hydrophilic side chain with positive charge. The Trp140Arg substitution would obviously lead to destabilization of the structure.

Four missense mutations were novel. Mutation in P14 (p.Leu205Ser) changed the conserved hydrophobic residue into a hydrophilic residue. Proline was involved in the mutations in P11 (p.Pro10Ser) and P17 (p.Ala208Pro). Proline is the only amino acid forming a ring with the backbone and bends the main chain of the protein in a characteristic way. Proline is a known breaker of secondary structures. The substitution in the two patients would profoundly change structure and functions of the protein. A p.Ala208Asp mutation was identified in an XHIGM patient and reported in 2002 [40].

Effects of the four novel point mutations were analyzed by using bioinformatics software PolyPhen-2 [41] and MutationTaster [42]. PolyPhen-2 predicted probably damaging effects to the protein with a score over 0.99 , respectively. MutationTaster predicts that mutations in P13, P14, and P17 were disease causing, with probability value over 0.98 .

Two patients had nonsense mutations at codon 236 and codon 218 , both of which were already reported [18, 36, 43]. Cys 218 was involved in formation of a disulfide bridge and nonsense mutation (p.C218X) had been reported in at least twelve XHIGM patients and one carrier, usually associated with more severe clinical presentation.

Nine patients had insertion/deletion mutations, all changing the reading frames and leading to large changes of the protein structure. P12 and P16 were from unrelated families and they had the same mutation (g.2091_2094delTAGA, c.158_161delTAGA, p.Ile53fsX13). This mutation had been reported in at least another 6 unrelated patients and was the fifth common mutation position in the CD40Lbase. The 4 deleted nucleotides were very close to the acceptor splice site of intron 1 and were flanked by repeat sequences, suggesting that these mutations might be caused by slipped mispairing mechanisms. The frame shift deletion led to a premature stop codon within the extracellular unique domain. P2 and P3 were cousins and they had the same mutation (g.186delG). The deletion in exon 1 led to a premature stop codon in the transmembrane domain.

In conclusion, we retrospectively investigated the clinical, immunological, and molecular features of 20 Chinese XHIGM patients diagnosed and followed up in our center. We identified 18 unique mutations in CD40L in these patients, with 12 novel mutations. Various mutations reflected the heterogeneity of CD40L gene, and we should go further to investigate the relationship between genotype and phenotype.

\section{Conflict of Interests}

None of the authors has any potential financial conflict of interests related to this paper. 


\section{Authors' Contribution}

Lin-Lin Wang and Wei Zhou are co-first authors and had equal contributions to the study.

\section{Acknowledgments}

The authors express their gratitude to all the patients and their families for their kindness for permission and cooperation. The authors are also grateful to Koon-Wing Chan, Department of Paediatrics and Adolescent Medicine, The University of Hong Kong, for his kind advice on sequence analysis.

\section{References}

[1] N. Qamar and R. L. Fuleihan, "The hyper IgM syndromes," Clinical Reviews in Allergy and Immunology, vol. 46, no. 2, pp. 120-130, 2014.

[2] A. R. Hayward, J. Levy, F. Facchetti et al., "Cholangiopathy and tumors of the pancreas, liver, and biliary tree in boys with X-linked immunodeficiency with hyper-IgM," The Journal of Immunology, vol. 158, no. 2, pp. 977-983, 1997.

[3] R. C. Allen, R. J. Armitage, M. E. Conley et al., "CD40 ligand gene defects responsible for X-linked hyper-IgM syndrome," Science, vol. 259, no. 5097, pp. 990-993, 1993.

[4] A. Aruffo, M. Farrington, D. Hollenbaugh et al., "The CD40 ligand, gp39, is defective in activated t cells from patients with X-linked hyper-IgM syndrome," Cell, vol. 72, no. 2, pp. 291-300, 1993.

[5] J. P. DiSanto, J. Y. Bonnefoy, J. F. Gauchat, A. Fischer, and G. de Saint Basile, "CD40 ligand mutations in X-linked immunodeficiency with hyper-IgM," Nature, vol. 361, no. 6412, pp. 541-543, 1993.

[6] R. Fuleihan, N. Ramesh, F. S. Rosen, and R. S. Geha, "Localization of the genetic defect in X-linked immunoglobulin deficiency with normal or elevated IgM (HIGMX-1) to the CD40 ligand gene," Transactions of the Association of American Physicians, vol. 106, pp. 91-95, 1993.

[7] U. Korthauer, D. Graf, H. W. Mages et al., "Defective expression of T-cell CD40 ligand causes X-linked immunodeficiency with hyper-IgM," Nature, vol. 361, no. 6412, pp. 539-541, 1993.

[8] A. C. Grammer, M. C. Bergman, Y. Miura, K. Fujita, L. S. Davis, and P. E. Lipsky, "The CD40 ligand expressed by human B cells costimulates B cell responses," The Journal of Immunology, vol. 154, no. 10, pp. 4996-5010, 1995.

[9] A. Chatzigeorgiou, M. Lyberi, G. Chatzilymperis, A. Nezos, and E. Kamper, "CD40/CD40L signaling and its implication in health and disease," BioFactors, vol. 35, no. 6, pp. 474-483, 2009.

[10] U. Schönbeck and P. Libby, "The CD40/CD154 receptor/ligand dyad," Cellular and Molecular Life Sciences, vol. 58, no. 1, pp. 443, 2001.

[11] G. Van Kooten and J. Banchereau, "CD40-CD40 ligand," Journal of Leukocyte Biology, vol. 67, no. 1, pp. 2-17, 2000.

[12] E. G. Davies and A. J. Thrasher, "Update on the hyper immunoglobulin M syndromes," British Journal of Haematology, vol. 149, no. 2, pp. 167-180, 2010.

[13] E. van Hoeyveld, P. X. Zhang, K. de Boeck, R. Fuleihan, and $\mathrm{X}$. Bossuyt, "Hyper-immunoglobulin M syndrome caused by a mutation in the promotor for CD40L," Immunology, vol. 120, no. 4, pp. 497-501, 2007.
[14] L. D. Notarangelo, M. Duse, and A. G. Ugazio, "Immunodeficiency with hyper-IgM (HIM)," Immunodeficiency Reviews, vol. 3, no. 2, pp. 101-121, 1991.

[15] L. D. Notarangelo, M. C. Peitsch, T. G. Abrahamsen et al., "CD40Lbase: a database of CD40L gene mutations causing Xlinked hyper-IgM syndrome," Immunology Today, vol. 17, no. 11, pp. 511-516, 1996.

[16] J. Levy, T. Espanol-Boren, C. Thomas et al., "Clinical spectrum of X-linked hyper-IgM syndrome," Journal of Pediatrics, vol. 131, part 1, no. 1, pp. 47-54, 1997.

[17] J. A. Winkelstein, M. C. Marino, H. Ochs et al., "The X-linked hyper-IgM syndrome: clinical and immunologic features of 79 patients," Medicine, vol. 82, no. 6, pp. 373-384, 2003.

[18] S. Nonoyama, M. Shimadzu, H. Toru et al., "Mutations of the CD40 ligand gene in 13 Japanese patients with X-linked hyperIgM syndrome," Human Genetics, vol. 99, no. 5, pp. 624-627, 1997.

[19] O. Cabral-Marques, S. Klaver, L. F. Schimke et al., "First report of the hyper-IgM syndrome registry of the latin American Society for Immunodeficiencies: novel mutations, unique infections, and outcomes," Journal of Clinical Immunology, vol. 34, no. 2, pp. 146-156, 2014.

[20] K. Seyama, S. Nonoyama, I. Gangsaas et al., "Mutations of the CD40 ligand gene and its effect on CD40 ligand expression in patients with X-linked hyper IgM syndrome," Blood, vol. 92, no. 7, pp. 2421-2434, 1998.

[21] Q. Lin, J. Rohrer, R. C. Allen et al., "A single strand conformation polymorphism study of CD40 ligand. Efficient mutation analysis and carrier detection for X-linked hyper IgM syndrome," The Journal of Clinical Investigation, vol. 97, no. 1, pp. 196-201, 1996.

[22] A. S. Levey and J. Coresh, "Chronic kidney disease," The Lancet, vol. 379, no. 9811, pp. 165-180, 2012.

[23] O. Cabral-Marques, L. Schimke, P. V. S. Pereira et al., "Expanding the clinical and genetic spectrum of human CD40L deficiency: the occurrence of paracoccidioidomycosis and other unusual infections in brazilian patients," The Journal of Clinical Immunology, vol. 32, no. 2, pp. 212-220, 2012.

[24] S. Norouzi, A. Aghamohammadi, S. Mamishi, S. D. Rosenzweig, and N. Rezaei, "Bacillus Calmette-Guérin (BCG) complications associated with primary immunodeficiency diseases," Journal of Infection, vol. 64, no. 6, pp. 543-554, 2012.

[25] W. J. Tang, Y. F. An, R. X. Dai et al., "Clinical, molecular, and T cell subset analyses in a small cohort of Chinese patients with hyper-IgM syndrome type 1," Human Immunology, vol. 75, no. 7, pp. 633-640, 2014.

[26] M. Madkaikar, M. Gupta, S. Chavan et al., "X-linked hyper IgM syndrome: clinical, immunological and molecular features in patients from India," Blood Cells, Molecules \& Diseases, 2014.

[27] Global Tuberculosis Report, “Global Tuberculosis Report 2013,” http://www.who.int/tb/publications/global_report/en/.

[28] T. Hayashi, S. P. Rao, P. R. Meylan, R. S. Kornbluth, and A. Catanzaro, "Role of CD40 ligand in Mycobacterium avium infection," Infection and Immunity, vol. 67, no. 7, pp. 3558-3565, 1999.

[29] G. M. Klug-Micu, S. Stenger, A. Sommer et al., "CD40 ligand and interferon- $\gamma$ induce an antimicrobial response against Mycobacterium tuberculosis in human monocytes," Immunology, vol. 139, no. 1, pp. 121-128, 2013.

[30] M. Carneiro-Sampaio and A. Coutinho, "Immunity to microbes: lessons from primary immunodeficiencies," Infection and Immunity, vol. 75, no. 4, pp. 1545-1555, 2007. 
[31] A. Jain, T. P. Atkinson, P. E. Lipsky, J. E. Slater, D. L. Nelson, and W. Strober, "Defects of T-cell effector function and postthymic maturation in X- linked hyper-IgM syndrome," Journal of Clinical Investigation, vol. 103, no. 8, pp. 1151-1158, 1999.

[32] W. Lee, T. R. Torgerson, M. J. Schumacher, L. Yel, Q. Zhu, and H. D. Ochs, "Molecular analysis of a large cohort of patients with the hyper immunoglobulin M (IgM) syndrome," Blood, vol. 105, no. 5, pp. 1881-1890, 2005.

[33] W. I. Lee, J. L. Huang, K. W. Yeh et al., "Clinical features and genetic analysis of Taiwanese patients with the hyper IgM syndrome phenotype," Pediatric Infectious Disease Journal, vol. 32, no. 9, pp. 1010-1016, 2013.

[34] A. Vargas-Hernández, L. Berrón-Ruiz, T. Staines-Boone et al., "Clinical and genetic analysis of patients with X-linked hyperIgM syndrome," Clinical Genetics, vol. 83, no. 6, pp. 585-587, 2013.

[35] M. A. García-Pérez, E. Paz-Artal, A. Corell et al., "Mutations of CD40 ligand in two patients with hyper-IgM syndrome," Immunobiology, vol. 207, no. 4, pp. 285-294, 2003.

[36] S. Weller, A. Faili, C. Garcia et al., "CD40-CD40L independent Ig gene hypermutation suggests a second B cell diversification pathway in humans," Proceedings of the National Academy of Sciences of the United States of America, vol. 98, no. 3, pp. 1166$1170,2001$.

[37] A. Villa, L. D. Notarangelo, J. P. Di Santo et al., "Organization of the human CD40L gene: implications for molecular defects in X chromosome-linked hyper-IgM syndrome and prenatal diagnosis," Proceedings of the National Academy of Sciences of the United States of America, vol. 91, no. 6, pp. 2110-2114, 1994.

[38] J. Thusberg and M. Vihinen, "The structural basis of hyper IgM deficiency-CD40L mutations," Protein Engineering, Design and Selection, vol. 20, no. 3, pp. 133-141, 2007.

[39] A. Aghamohammadi, N. Parvaneh, N. Rezaei et al., "Clinical and laboratory findings in Hyper-IgM syndrome with novel CD40L and AICDA mutations," Journal of Clinical Immunology, vol. 29, no. 6, pp. 769-776, 2009.

[40] H. Piirilä, J. Väliaho, and M. Vihinen, "Immunodeficiency mutation databases (IDbases)," Human Mutation, vol. 27, no. 12, pp. 1200-1208, 2006.

[41] I. A. Adzhubei, S. Schmidt, L. Peshkin et al., "A method and server for predicting damaging missense mutations," Nature Methods, vol. 7, no. 4, pp. 248-249, 2010.

[42] J. M. Schwarz, C. Rödelsperger, M. Schuelke, and D. Seelow, "MutationTaster evaluates disease-causing potential of sequence alterations," Nature Methods, vol. 7, no. 8, pp. 575-576, 2010.

[43] S. Danielian, M. Oleastro, M. Eva Rivas, C. Cantisano, and M. Zelazko, "Clinical follow-up of 11 Argentinian CD40Ldeficient patients with 7 unique mutations including the socalled "milder" mutants," Journal of Clinical Immunology, vol. 27, no. 4, pp. 455-459, 2007.

[44] K. C. Gilmour, D. Walshe, S. Heath et al., "Immunological and genetic analysis of 65 patients with a clinical suspicion of $\mathrm{X}$ linked hyper-IgM," Molecular Pathology, vol. 56, no. 5, pp. 256262, 2003.

[45] A. Rangel-Santos, V. L. Wakim, C. M. Jacob et al., "Molecular characterization of patients with X-linked Hyper-IgM syndrome: description of two novel CD40L mutations," Scandinavian Journal of Immunology, vol. 69, no. 2, pp. 169-173, 2009.

[46] CD40Lbase, 2011, http://structure.bmc.lu.se/idbase/CD40Lbase/ index.php. 


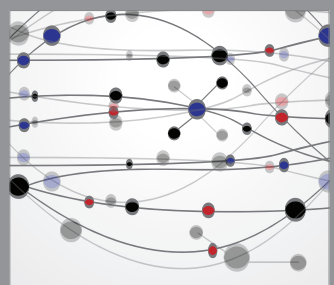

The Scientific World Journal
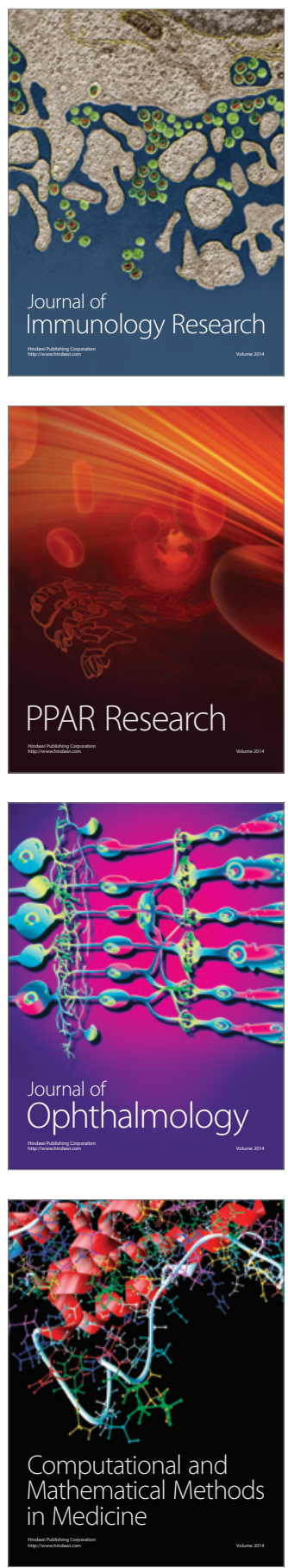

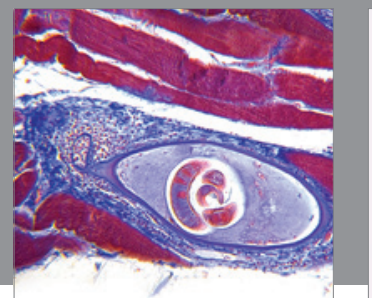

Gastroenterology

Research and Practice
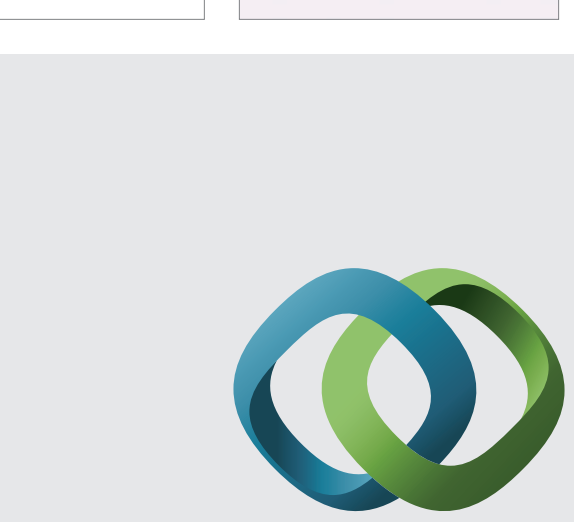

\section{Hindawi}

Submit your manuscripts at

http://www.hindawi.com
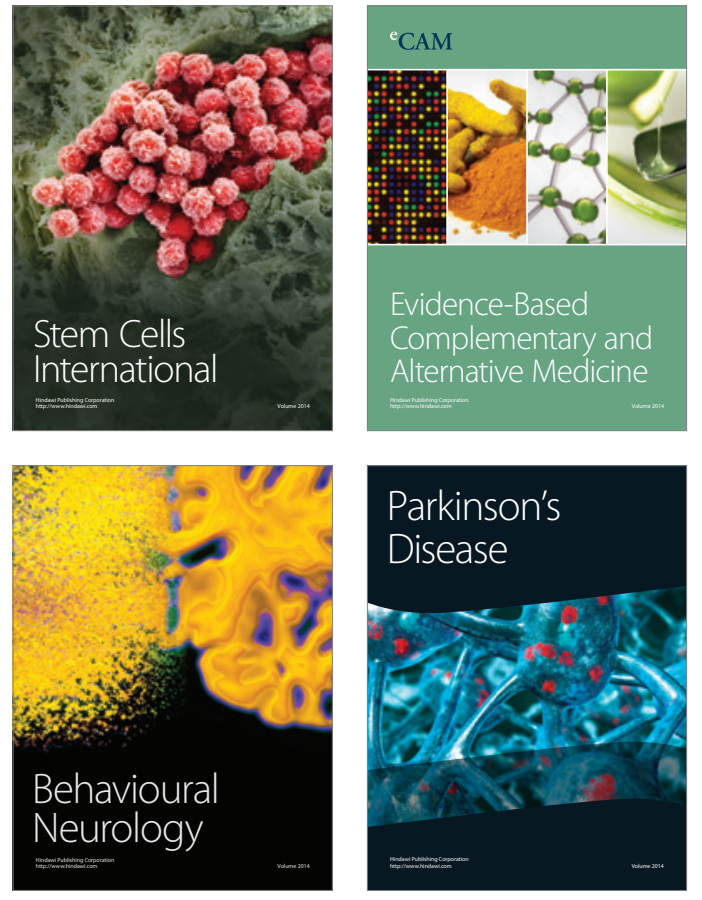
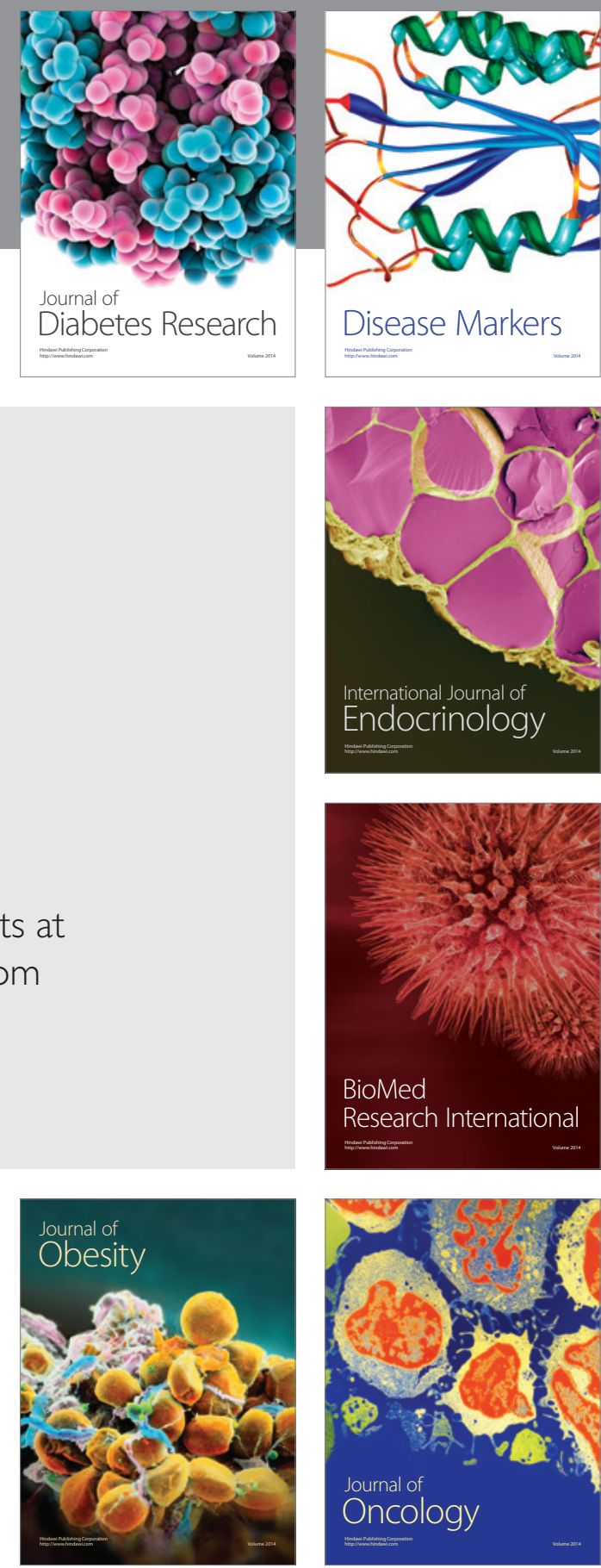

Disease Markers
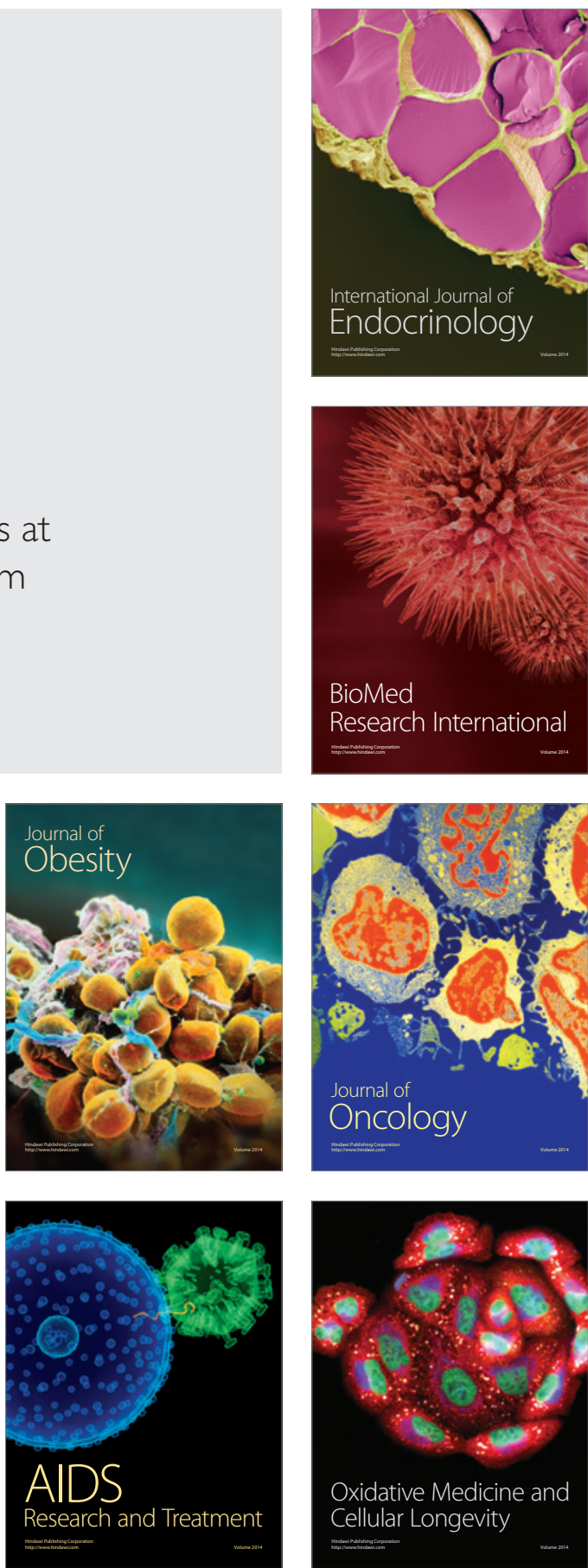